\title{
Conservative surgical management for immediate postpartum hemorrhage
}

\author{
Pimpitcha Puangsricharoen, Tarinee Manchana*
}

\begin{abstract}
Background: Conservative surgical management for postpartum hemorrhage (PPH), such as balloon tamponade, uterine compression suture, and uterine artery ligation, has the benefit of preserving reproductive function.

Objectives: To assess the efficacy and subsequent pregnancy outcome of conservative surgical management for patients with immediate PPH.

Methods: Medical records of patients who had PPH between January 2011 and December 2016 were reviewed. Conservative surgical management included B-Lynch uterine compression suture, Bakri balloon tamponade, and uterine artery ligation. The treatments were considered successful if patients did not require subsequent hysterectomy. Perioperative complications and subsequent pregnancy outcomes were recorded.

Results: Of 30,271 deliveries, 669 patients experienced PPH or 2.2\% of total deliveries. Sixty-one patients (9.1\%) did not respond to medical treatment with various uterotonic agents. Hysterectomy was selected initially in 30 patients. Conservative surgical management was performed in 31 patients: 15 Bakri balloon tamponade, 13 uterine compression suture, and 3 uterine artery ligation. There were 3 patients who failed Bakri balloon tamponade and proceeded to perform uterine compression suture with successful outcome. The success rates for conservative surgical treatment were $66.7 \%, 75 \%$, and $66.7 \%$, respectively. All patients who had successful conservative surgical management resumed normal menstruation. Three out of 11 patients $(27.3 \%)$ who desired subsequent pregnancy were able to conceive and carry out a viable pregnancy.

Conclusion: Conservative surgical management has acceptable success rates for controlling intractable immediate PPH. Implementation of such procedures should be done to preserve fertility and decrease maternal morbidity and mortality.
\end{abstract}

Keywords: balloon occlusion; postpartum hemorrhage; uterine artery ligation; uterine compression suture

Postpartum hemorrhage $(\mathrm{PPH})$ remains the leading cause of maternal morbidity and mortality worldwide, especially in developing countries [1]. In Asia, PPH accounts for 30.8\% of all direct obstetric mortalities [2]. Common cause of PPH is uterine atony. This condition can usually be managed with medical treatment using uterotonic agents. However, medical treatment may be unsuccessful in some patients, and these patients require further surgical intervention. Hysterectomy is a common surgical procedure for such situation, but it is not suitable for patients who desire to maintain fertility. Conservative surgical management such as balloon tamponade, uterine compression suture, and uterine artery ligation has the benefit

*Correspondence to: Tarinee Manchana, Department of Obstetrics and Gynecology, Faculty of Medicine, Chulalongkorn University and King Chulalongkorn Memorial Hospital, Bangkok 10330, Thailand, e-mail: Tarinee.m@chula.ac.th

Department of Obstetrics and Gynecology, Faculty of Medicine, Chulalongkorn University and King Chulalongkorn Memorial Hospital, Bangkok 10330, Thailand

O Open Access. (c) 2019 Puangsricharoen and Manchana, published by Sciendo. (cc)BY-NC-ND This work is licensed under the Creative Commons Attribution NonCommercial-NoDerivatives 4.0 License. 
of preserving reproductive function. The Bakri balloon tamponade is effective at controlling bleeding from the placental site in the lower uterine segment [3]. In 1997, B-Lynch uterine compression suture was introduced [4]. Various studies have reported good surgical outcomes with few complications [5-7]. Uterine artery ligation was first described by Howard Kelly for the treatment of intraoperative bleeding in cervical cancer patients prior to its application in PPH [5]. This method is recommended for the treatment of PPH caused by uterine atony if other conservative methods have failed [6].

In Thailand, there were few studies on the efficacy of conservative surgical management for PPH. However, limited study has evaluated the long-term fertility following these procedures $[7,8]$. Our study aims to report on the efficacy and subsequent pregnancy outcomes in patients with intractable immediate PPH who had successful conservative surgical management.

\section{Materials and methods}

This study was approved by the Institutional Review Board of the Faculty of Medicine, Chulalongkorn University, Bangkok, Thailand (certificate of approval no.920/2017).

The medical records of patients who had immediate PPH between January 2011 and December 2016 were reviewed. PPH is defined as blood loss of more than $500 \mathrm{ml}$ following vaginal delivery and $1,000 \mathrm{ml}$ following cesarean delivery. Conservative surgical management included B-Lynch uterine compression suture, Bakri balloon tamponade, and uterine artery ligation. Demographic data such as age, parity, gestation of pregnancy at delivery, and cause of PPH were recorded. The operative outcomes, intraoperative and perioperative complications, and need for subsequent hysterectomy were recorded.

The standard protocols of uterotonic agents for controlling PPH at the King Chulalongkorn Memorial Hospital were oxytocin infusion $20-40 \mathrm{IU}$ in $1,000 \mathrm{ml} 0.9 \% \mathrm{NaCl}$; methylergometrine $0.2 \mathrm{mg}$ intravenous, repeated every $15 \mathrm{~min}$ to a maximum dose of $1 \mathrm{mg}$; and sulprostone $500 \mu \mathrm{mg}$ intravenous drip for $1 \mathrm{~h}$. If the bleeding continued, conservative surgical management or hysterectomy was chosen according to the patient's preference and surgeon's judgment. The Bakri balloon tamponade was filled with $300-500 \mathrm{ml}$ saline and placed in the uterine cavity for $24-48 \mathrm{~h}$. Uterine compression suture was performed as described by Allam and B-Lynch [9].

The conservative surgical treatment was considered successful if the patient did not require subsequent hysterectomy. For the patients with successful conservative surgical treatments, postoperative complications at 6 weeks postpartum were collected from the medical records and subsequent pregnancy outcomes were acquired via direct contact by telephone.

\section{Results}

Of 30,271 deliveries, 669 patients experienced immediate $\mathrm{PPH}$, or $2.2 \%$ of total deliveries. Most patients had successful treatment with uterotonic agents, and only 61 patients $(9.1 \%)$ did not respond to the medical treatment. Hysterectomy was selected initially for 30 patients $(49.2 \%)$. Conservative surgical management was performed in 31 patients $(50.8 \%)$. The demographic data are shown in Table 1. Mean age of the patients was 33 years, ranged between 20 and 41 years. Twenty-one patients $(67.7 \%)$ delivered at term gestation. Emergency cesarean delivery was the most common route of delivery.

Table 1. Demographic data

\begin{tabular}{|c|c|c|}
\hline Patient Characteristics & Number of patients & Percent \\
\hline Primigravida & 13 & 41.9 \\
\hline Multigravida & 18 & 58.1 \\
\hline \multicolumn{3}{|l|}{ Gestational age } \\
\hline $28-36$ weeks & 10 & 32.3 \\
\hline $37-42$ weeks & 21 & 67.7 \\
\hline \multicolumn{3}{|l|}{ Route of delivery } \\
\hline Vaginal delivery & 3 & 9.7 \\
\hline Elective cesarean delivery & 9 & 29 \\
\hline Emergency cesarean delivery & 19 & 61.3 \\
\hline \multicolumn{3}{|l|}{ Indication for cesarean delivery } \\
\hline Cephalopelvic disproportion & 9 & 32.1 \\
\hline Placenta previa & 9 & 32.1 \\
\hline Previous cesarean delivery & 3 & 14.3 \\
\hline Multiple pregnancy & 2 & 10.7 \\
\hline Breech presentation & 4 & 7.1 \\
\hline Cervical mass & 1 & 3.6 \\
\hline \multicolumn{3}{|l|}{ Birth weight (g) } \\
\hline$<2,500$ & 8 & 25.8 \\
\hline $2,500-3,500$ & 18 & 58.1 \\
\hline $3,501-4,000$ & 3 & 9.7 \\
\hline$>4,000$ & 2 & 6.4 \\
\hline \multicolumn{3}{|l|}{ Cause of postpartum hemorrhage } \\
\hline Uterine atony & 23 & 74.2 \\
\hline Placenta previa & 2 & 6.5 \\
\hline Bleeding from placental bed & 6 & 19.4 \\
\hline \multicolumn{3}{|l|}{$\begin{array}{l}\text { Type of conservative surgical } \\
\text { management }\end{array}$} \\
\hline Bakri balloon tamponade & 15 & 48.4 \\
\hline B-Lynch uterine compression suture & 13 & 41.9 \\
\hline Uterine artery ligation & 3 & 9.7 \\
\hline
\end{tabular}


Indications for cesarean delivery included cepholopelvic disproportion (CPD), placenta previa, and previous cesarean delivery. The most common cause of PPH was uterine atony, accounting for $74.2 \%$ of patients, followed by bleeding from the placental bed without evidence of placenta previa (19.4\%) and placenta previa $(6.5 \%)$.

B-Lynch uterine compression suture was performed in $13 \mathrm{PPH}$ patients due to uterine atony. The Bakri balloon tamponade was performed in 15 patients. Three patients had continued bleeding after Bakri balloon tamponade, and B-Lynch uterine compression suture was applied. Uterine artery ligation was done in 3 patients. The surgical outcomes are shown in Table 2. There were no maternal deaths in this series; however, disseminated intravascular coagulopathy (DIC) was detected in 8 patients $(25.8 \%)$ : 5 patients in the Bakri balloon tamponade group and 3 patients in the uterine compression suture group. Massive blood transfusion was defined as receiving packed red blood cells (PRC) of 10 units or more [10]. Only 1 patient in the Bakri balloon group (3.2\%) required a massive blood transfusion and developed pulmonary edema. Intra-abdominal injuries such as bladder and bowel injury were reported during cesarean delivery in 4 patients in the Bakri balloon tamponade and uterine compression suture groups.

Hysterectomy could not be avoided in $8 \mathrm{PPH}$ patients $(25.8 \%)-4$ in the Bakri balloon tamponade group, 3 in the B-Lynch uterine compression suture group, and 1 in the uterine artery ligation group. Two patients were primigravida. Three patients performed elective cesarean sections, and the other 5 patients performed emergency cesarean sections. Success rates were $73.3 \%, 76.9 \%$, and $66.7 \%$ for Bakri balloon tamponade, B-Lynch uterine compression suture, and uterine artery ligation, respectively. Per protocol analysis, there were 3 patients who failed Bakri balloon tamponade and preceded to perform uterine compression suture, and the success rate of uterine compression suture was $75 \%$ (12/16 patients) but $66.7 \%$ ( $8 / 12$ patients) for Bakri balloon tamponade.

All 23 patients who had successful conservative surgical management reported normal menstruation after the procedures. Eleven of 23 patients desired subsequent pregnancy. Three of them $(27.3 \%), 1$ from each conservative surgical treatment group, were able to conceive and carry out viable pregnancy within 1 year.

\section{Discussion}

Incidence of immediate PPH remains high and tends to increase with increasing cesarean section rates. Cesarean delivery, particularly emergency cesarean delivery, is associated with a higher risk of PPH [11]. PPH is problematic and leads to maternal morbidity and mortality worldwide. Uterine atony is the most common cause of $\mathrm{PPH}$, and it is usually managed with uterotonic agents. If medical treatments are unsuccessful, conservative surgical management such as uterine compression suture, balloon tamponade, and uterine artery ligation are considered especially in patients who desire to preserve their fertility function. The incidence of PPH in our study is concordant with the findings from other countries in Asia. In 2012, Calvert et al. reported an incidence of $1.9 \%$ in Asia, including Japan, Hong Kong, East Asia, and Thailand [12]. Another study done in Thailand showed a $4.2 \%$ incidence rate of PPH [9]. Different definitions of PPH and methods for blood loss assessment might be reasons for different PPH incidence rates [13].

Table 2. Outcomes of conservative surgical treatments

\begin{tabular}{|c|c|c|c|}
\hline & $\begin{array}{l}\text { Bakri balloon tamponade } \\
\qquad(n=15)\end{array}$ & $\begin{array}{l}\text { B-Lynch uterine compression suture } \\
\qquad(n=13)\end{array}$ & $\begin{array}{l}\text { Uterine artery ligation } \\
\qquad(n=3)\end{array}$ \\
\hline Estimated blood loss, ml (mean \pm SD) & $3,346 \pm 2,647 \mathrm{ml}$ & $2,984 \pm 2,540 \mathrm{ml}$ & $2,133 \pm 1,001 \mathrm{ml}$ \\
\hline \multicolumn{4}{|l|}{ Blood component, units (mean \pm SD) } \\
\hline Packed red cells & $8 \pm 5$ & $8 \pm 3$ & $2 \pm 3$ \\
\hline Fresh frozen plasma & $7 \pm 3$ & $5 \pm 3$ & $1 \pm 4$ \\
\hline Platelets & $5 \pm 5$ & $2 \pm 5$ & 0 \\
\hline \multicolumn{4}{|l|}{ Perioperative complications } \\
\hline Disseminated intravascular coagulopathy & $5(33.3 \%)$ & $3(23.1 \%)$ & 0 \\
\hline Bladder injury & $1(6.7 \%)$ & $1(7.7 \%)$ & 0 \\
\hline Bowel injury & $1(6.7 \%)$ & $1(7.7 \%)$ & 0 \\
\hline Hospital stay (days) (mean \pm SD) & $15 \pm 7$ & $13 \pm 5.9$ & $3 \pm 10$ \\
\hline Failure rate (conversion to hysterectomy) & $4(26.7 \%)$ & $3(23.1 \%)$ & $1(33.3 \%)$ \\
\hline Subsequent pregnancy in patients who desired & $25 \%(1 / 4)$ & $20 \%(1 / 5)$ & $50 \%(1 / 2)$ \\
\hline
\end{tabular}


The decisions to perform conservative surgical treatments were made according to the patient's preference and the surgeon's professional judgment. Bakri balloon had been reported to achieve hemostasis in PPH related to placental bed bleeding from low-lying placenta [3]. However, it is used not only for the bleeding from the lower segment but also for uterine atony. The uterine compression suture used in our institute was the B-Lynch technique, which has been reported to be effective in the treatment of PPH due to uterine atony. Correct application of the B-Lynch suture distributes tension across the uterine cavity and maintains the uterine tone until involution starts [14]. Some modifications to the compression suture, such as Hayman's and Cho's, have been described. The potential setbacks of modified sutures include occlusion of the uterine cavity and retention of blood clots, both of which can interfere with the physiological postpartum involution of the uterus $[15,16]$. However, which compression suture techniques is the most effective is unknown because there was no comparative study [17].

A systematic review showed no statistically significant differences in the efficacy of uterine compression suture, balloon tamponade, iliac artery ligation (IAL), and arterial embolization; the success rates were reported as $84 \%$ for balloon tamponade, $91.7 \%$ for uterine compression suture, and $84.6 \%$ for IAL or uterine devascularization [18]. In our study, the Bakri balloon tamponade and uterine compression suture had similar success rates $(66.7 \%$ and $75 \%$, respectively), which were slightly lower than the study by Kaya et al. (79.1\% and $80 \%$ ) [19]. The success rates were higher when combined with IAL, 95\% in the Bakri balloon tamponade group and $91.6 \%$ in the B-Lynch group. One previous study in Thailand reported the success rate of uterine compression suture to be $95.8 \%$ (23 out of 24 patients). In 6 of them, combination of B-Lynch suture and bilateral uterine artery ligation was performed [8]. Mean estimated blood loss in our study was slightly higher than previous reports. More severity and delayed to perform conservative surgical management might led to lower success rates in our study. In the uterine artery ligation group, hemorrhage could be controlled in 2 out of 3 patients (66.7\%). A study from Boynukalin et al. reported a success rate of 20 out of 26 patients (76.9\%) [20]. Due to the limited number of patients in our study, more patients are needed to confirm the efficacy of this approach.

The impact of conservative surgical treatment on subsequent pregnancy outcome is interesting. Doumouchtsis et al. reported that it did not involve the menstrual and fertility outcomes in most women. The authors reported that $91.2 \%$ women experienced menstruation within 6 months after surgery. Seventy-seven percent of women who desired another pregnancy achieved conception [17]. Resumption of normal menstruation was noted in all patients in our report. However, the subsequent pregnancy rate was lower in our study when compared with previous studies $[17,21,22]$. Longer follow-up is needed to confirm the exact pregnancy rates.

\section{Conclusion}

Conservative surgical management is effective for the treatment of intractable immediate PPH, with acceptable success rates and low complication rates. Such procedures tend to have promising fertility outcomes. Medical providers should be encouraged to utilize these treatments without delay. Timely recognition and prompt intervention play a crucial role in preventing maternal morbidity and mortality.

Author contributions. Both authors made substantial contributions to the conception and design of the study, and acquisition of the data. PP substantially analyzed and interpreted the data. Both authors drafted the manuscript and TM critically revised it. Both authors approved the final version submitted for publication and take responsibility for the statements made in the published article.

Conflict of interest statement. The authors have each completed and submitted an International Committee of Medical Journal Editors Uniform Disclosure Form for Potential Conflicts of Interest. Neither of the authors has any potential conflict of interest to disclose.

\section{References}

[1] ACOG Practice Bulletin: Clinical management guidelines for obstetrician-gynecologists number 76, October 2006: postpartum hemorrhage. Obstet Gynecol. 2006; 108:1039-47.

[2] Khan KS, Wojdyla D, Say L, Gulmezoglu AM, Van Look PF. WHO analysis of causes of maternal death: a systematic review. Lancet. 2006; 367:1066-74

[3] Bakri YN, Amri A, Abdul Jabbar F. Tamponade-balloon for obstetrical bleeding. Int J Gynaecol Obstet. 2001; 74:139-42.

[4] B-Lynch C, Coker A, Lawal AH, Abu J, Cowen MJ. The B-Lynch surgical technique for the control of massive postpartum haemorrhage: an alternative to hysterectomy? Five cases reported. Br J Obstet Gynaecol. 1997; 104:372-5.

[5] Siegel P, Mengert WF. Internal iliac artery ligation in obstetrics and gynecology. JAMA. 1961; 178:1059-62.

[6] World Health Organization. WHO recommendations for the prevention and treatment of postpartum haemorrhage [online]. 2012 [cited 2018 Oct 10]. Available from: https://apps.who.int/iris/ bitstream/handle/10665/75411/9789241548502_eng.pdf;jsessionid=8E2 8469608D102039DFE69D28C4E76EB? sequence $=1$.

[7] Charoendumrongsup P. B lynch suturing in uterine atony during ceasarean section. Reg 11 Med J. 2015; 29:189-93. 
[8] Kaoiean S. Successful use of the B-Lynch uterine compression suture in treating intractable postpartum hemorrhage after cesarean delivery in Rajavithi Hospital. J Med Assoc Thai. 2013; 96:1408-15.

[9] Allam MS, B-Lynch C. The B-Lynch and other uterine compression suture techniques. Int J Gynaecol Obstet. 2005; 89:236-41.

[10] Shaz BH, Dente CJ, Harris RS, MacLeod JB, Hillyer CD. Transfusion management of trauma patients. Anesth Analg. 2009; 108:1760-8.

[11] Fawcus S, Moodley J. Postpartum haemorrhage associated with caesarean section and caesarean hysterectomy. Best Pract Res Clin Obstet Gynaecol. 2013; 27(2):233-49.

[12] Calvert C, Thomas SL, Ronsmans C, Wagner KS, Adler AJ, Filippi V. Identifying regional variation in the prevalence of postpartum haemorrhage: a systematic review and meta-analysis. PLoS One. 2012; 7:e41114.

[13] Carroli G, Cuesta C, Abalos E, Gulmezoglu AM. Epidemiology of postpartum haemorrhage: a systematic review. Best Pract Res Clin Obstet Gynaecol. 2008; 22:999-1012.

[14] B-Lynch C. Partial ischemic necrosis of the uterus following a uterine brace compression suture. BJOG. 2005; 112:126-7.

[15] Hayman RG, Arulkumaran S, Steer PJ. Uterine compression sutures: surgical management of postpartum hemorrhage. Obstet Gynecol. 2002; 99:502-6.

[16] Cho JH, Jun HS, Lee CN. Hemostatic suturing technique for uterine bleeding during cesarean delivery. Obstet Gynecol. 2000; 96:129-31.
[17] Doumouchtsis SK, Nikolopoulos K, Talaulikar V, Krishna A, Arulkumaran S. Menstrual and fertility outcomes following the surgical management of postpartum haemorrhage: a systematic review. BJOG. 2014; 121:382-8.

[18] Doumouchtsis SK, Papageorghiou AT, Arulkumaran S. Systematic review of conservative management of postpartum hemorrhage: what to do when medical treatment fails. Obstet Gynecol Surv. 2007; 62:540-7.

[19] Kaya B, Guralp O, Tuten A, Unal O, Celik MO, Dogan A. Which uterine sparing technique should be used for uterine atony during cesarean section? The Bakri balloon or the B-Lynch suture? Arch Gynecol Obstet. 2016; 294:511-7.

[20] Boynukalin FK, Boyar H, Gormus H, Aral AI, Boyar N. Bilateral hypogastric artery ligation in emergency setting for intractable postpartum hemorrhage: a secondary care center experience. Clin Exp Obstet Gynecol. 2013; 40:85-8.

[21] Tsitlakidis C, Alalade A, Danso D, B-Lynch C. Ten year follow-up of the effect of the B-Lynch uterine compression suture for massive postpartum hemorrhage. Int J Fertil Womens Med. 2006; 51:262-5.

[22] El-Hamamy E, B-Lynch C. A worldwide review of the uses of the uterine compression suture techniques as alternative to hysterectomy in the management of severe post-partum haemorrhage. J Obstet Gynaecol. 2005; 25:143-9. 